\section{PARENTAL PERCEPTIONS ON RISK OF CHILD INJURIES IN THE COLOMBO DISTRICT OF SRI LANKA}

${ }^{1}$ Indrakantha Welgama, ${ }^{2}$ Hemantha Herath, ${ }^{3}$ Samath Dharmaratne. ${ }^{1}$ Office of the Regional Director of Health Services, Colombo, Sri Lanka; ${ }^{2}$ Ministry of Health, Sri Lanka; ${ }^{3}$ University of Peradeniya, Sri Lanka

\subsection{6/injuryprev-2016-042156.458}

Background The objective of this study was to describe parental perceived factors which may influence the occurrence of unintentional traumatic injuries among children aged 5 to 12 years, in the Colombo district of Sri Lanka.

Methods A community based qualitative study consisting of several Focus Group Discussions (FGD), were conducted, covering four separate areas of the district. The study population was housewives, who were parents of children. Each FGD consisted of 5 participants, a facilitator and a note taker. They were conducted in a private setting within their villages.

Results The study showed that home, schools, playgrounds and roads were the most common injury risk environments according to the parental perception. Risk factors for home injuries were hyperactive children, non-vigilant parents, socio-economic family factors and behaviour of other siblings. Unruly students, inexperienced teachers and unsafe school environment were identified to be the risks at school. Playground conditions and incorrect techniques in sports were causes for playground injuries. Travelling in 2-wheel and 3-wheel vehicles were a risk factor for road accidents. Most parents thought inexperience of children were a main cause for sustaining injuries. Lack of proper parental guidance in carrying out activities put children at risk of injury. Parental awareness of child's friends, their behaviour and activities were found to be important. Knowledge of first aid in the children and proper implementation of regulations by the authorities were also mentioned as important steps that could minimise the occurrence, and effects of injuries.

Conclusions Parents have a responsibility in the prevention of injuries of their children. They need to teach and train their children to identify injury risks factors and avoid them or act safely. They need to be aware of the child's friends, their behaviour and must have a close bond with their children to prevent them from injuries.

\section{NURSES' PERCEPTIONS ABOUT CHILD ABUSE}

${ }^{1}$ Ahmad Saifan, ${ }^{2}$ Intima Alrimawi, ${ }^{3}$ Ibraheem Bashayreh. ${ }^{1}$ Applied Science Private University; ${ }^{2}$ Birzeit University; ${ }^{3}$ Philadelphia University

10.1136/injuryprev-2016-042156.459

Background Despite the efforts to protect children around the world, child abuse and neglect remain serious and global problems. In Palestine, child abuse is hidden under the community culture, does not appear in the Ministry of Health official reports, and little is known about nurses' perceptions towards this phenomenon. The aim of this study was to identify nurses' perceptions about child abuse definition, whether they faced such cases during their work, and how they managed them.
Methods Data were collected using descriptive survey (exploratory) approach. A total of 84 nurses from a major hospital in Ramallah city in Palestine were surveyed.

Results Only 33\% of the participants intend to use referral system in co-operation with the Ministry of Social Affairs, child protection organisations, or the police in Palestine to deal with child abuse cases. The most seen abuse case was neglect (79\%), followed by psycho-logical abuse (61\%), then physical abuse (57\%) and the least seen was sexual abuse (27\%).

Conclusions Most of the participants do not know how to deal with child abuse effectively. This research provides baseline information for understanding nurses' practice in the ground, and it helps in presenting the appropriate conditions that enable nurses to fully practice their role toward such cases.

\section{CHARACTERISTICS AND RISK FACTORS OF DOG- INDUCED INJURY AMONG CHILDREN IN TWO DIFFERENT SIZE CITIES OF CHINA}

Ying Chen, Liping Li. Centre for Injury Prevention Research, Shantou University Medical College, Shantou, China

\subsection{6/injuryprev-2016-042156.460}

Background Dogs are a potential source of several health hazards for humans. Global public attention has recently focused on bites and other canine aggression, but few data in China. Our study aims to estimate the prevalence of dog-induced injuries and to identify possible risk factors in the two cities with different population and development level.

Methods A cross-sectional survey of 9380 children from middle size city, Shantou and super city, Shenzhen in Guangdong province, was conducted in 2015. A self-administered questionnaires was used to collect participant's information on socio-demographics and injury occurrence. Multivariate logistic regression was used to assess the individual and family factors associated with odds of dog-induced injuries.

Results Among 9380 children, the range of age was 6-20 $(12.85 \pm 3.29)$, and $50.5 \%$ were male. 1413 (15.1\%) children reported they had been injured by $\mathrm{dog}$, In the two areas, $18.1 \%$ of children had been injured by dog in Shenzhen and $11.7 \%$ was attacked in Shantou $\left(\mathrm{x}^{2}=75.03, P<0.01\right)$. Multivariate logistic regression results for all children showed dog-induced injury was associated with middle-school age (OR: 2.17, 95\% CI: 1.712.74), living in rural area (OR: $2.48,95 \% \mathrm{CI}: 1.93-3.19)$, being fond of animals (OR: 1.46, 95\% CI: 1.09-1.91), poor academic performance (OR:1.54, 95\% CI: 1.28-1.85), both parents working outside (OR: 1.42, 95\% CI: 1.17-1.72) and dog owner (OR: 1.76, 95\% CI: 1.40-2.21). The dog-induced injury rate was no significant difference among boys and girls. Moreover, hierarchical multiple regression analysis were conducted by different city, showed increased risk of non-only child, poor academic performance and dog owner were significant only in Shantou (middle size city), while middle-school age, living in rural area and mother working outside only in Shenzhen(super city).

Conclusions Dog-induced injuries have become a pressing public health problem among Chinese children. Strategies targeting the risk factors may be effective for the prevention of injuries by 
dogs. We should pay more attention to left-behind children in rural areas who parents working outside to big cities.

\section{ASSOCIATION BETWEEN TEMPERAMENT AND FRACTURE RISK IN PRESCHOOL-AGE CHILDREN: A CASE CONTROL STUDY}

${ }^{1}$ Kandace Ryckman, 1,2,3 Sarah A Richmond 11 Aura Anderson, ${ }^{1}$ Catherine Birken, ${ }^{1}$ Patricia Parkin, ${ }^{1}$ Colin Macarthur, ${ }^{4}$ Jonathon Maguire, ${ }^{1}$ Andrew Howard. ${ }^{1}$ Hospital for Sick Children, Canada; ${ }^{2}$ York University, Canada; ${ }^{3}$ University of Calgary, Canada; ${ }^{4}$ University of Toronto, Canada

\subsection{6/injuryprev-2016-042156.461}

Background Approximately half of all children will sustain a fracture during childhood and adolescence. Understanding the factors that place a child at increased risk of fracture is necessary to inform effective injury prevention strategies.

Methods A case-control study design with 128 cases and 426 frequency-matched controls (age and sex) was used to examine the risk of fracture (radiologist diagnosed fracture) in children ages 3-6 years. Cases were recruited from the Hospital for Sick Children Fracture Clinic and controls from the TARGet Kids primary care paediatric network. The Childhood Behaviour Questionnaire (CBQ), a 36-item caregiver response questionnaire was used to assess three temperament factors: surgency (i.e. impulsivity, high activity level); negative affect (i.e. anger, fear, discomfort); and effortful control (i.e. inhibitory control, attentional focusing) as the exposure.

Results Unadjusted logistic models demonstrated no association between children with a fracture and higher scores of surgency (unadjusted OR $=1.06,95 \% \mathrm{CI}: 0.84,1.34$ ), negative affect (unadjusted OR $=1.15,95 \%$ CI: $0.93,1.42$ ) or effortful control (unadjusted OR $=0.80,95 \% \mathrm{CI}: 0.63,1.03$ ). Models adjusted for covariates (waist circumference, maternal education, history of fracture, attendance in daycare, amount of time spent in unstructured playtime outdoors, having been breastfed, daily milk and soda consumption), also demonstrated no significant association with surgency $(\mathrm{OR}=1.00,95 \% \mathrm{CI}: 0.78,1.29)$, negative affect $(\mathrm{OR}=1.09,95 \% \mathrm{CI}: 0.86,1.37)$ and effortful control $(\mathrm{OR}=0.80,95 \% \mathrm{CI}: 0.61,1.05)$.

Conclusions Children who are more extraverted, impulsive, aggressive or easily distracted as measured by the CBQ, are not at greater odds of experiencing a fracture, compared to their peers.

\section{CO-DESIGN PROJECT TO RAISE AWARENESS AROUND SEXUAL VIOLENCE AND SAFETY IN AUCKLAND YOUTH}

Kristin Good. Accident Compensation Corporation and Auckland District Health Board, Auckland, New Zealand

\subsection{6/injuryprev-2016-042156.462}

Background New Zealand has one of the highest rates of child sexual violence in the developed world. One in four girls are sexually abused before the age of 15 . Maori and Pacific Island girls are at significantly increased risk compared to European girls. Most abuse occurs at home by someone that is known to them. One in seven boys may be sexually abused before adulthood. The average age of abuse is nine.

Long-term effects of sexual violence on children can be significant. Untreated, there is a strong correlation with mental health disorders and lifelong social problems. Cultural differences influence the way youth interpret and respond to sexual violence. Social and economic costs are high.

Methods Youth with lived experience were involved in co-designing a Youth Innovation Forum with the local District Health Board and Accident Compensation Corporation. Sexual violence was a focus of the day. Novel ways of communicating through youth were used, including music and theatre, to create a powerful message raising awareness. Social media was an important tool. Cultural aspects were important in designing the final product. Psychological support was available on the day to ensure safety.

Results The creative intervention was powerful. It became the focal point of the day. Following the presentation a number of youth came forward seeking help for themselves and others. Psychological support was essential to ensure safety. Feedback confirmed the value of the presentation in increasing awareness and understanding. Repeat performances and a video presentation have spread the message to wider audiences. The success of the day has ensured funding for the event next year.

Conclusions Co-design ensured the message was relevant to youth. Including youth in delivering the message increased the relevance to the audience. Social media was effective in supporting the campaign. Psychological support is essential when delivering a powerful message to a potentially vulnerable audience.

\section{A RESEARCH ON E-DEVICES AND WALKING AMONG TEENS IN SHANGHAI: HOW THEY DO AND WHAT THEY SAY}

${ }^{1}$ Fannie Wang, ${ }^{2}$ Yan $Y u,{ }^{1,3}$ Mulder Wang, ${ }^{1}$ Xinyi Hou, ${ }^{2}$ Monica Cui, ${ }^{4} J u a n j u a n$ Peng ${ }^{4}$ Wenjuan Jiang. 'Safe Kids China, Shanghai; ${ }^{2}$ Shanghai CDC, Shanghai; ${ }^{3}$ Shanghai Traffic Police, Shanghai; ${ }^{4}$ Shanghai Education Commission, Shanghai

\subsection{6/injuryprev-2016-042156.463}

Background Road traffic accidents is the first killer to teens aged 15-17 worldwide. In China, the same trend happens on teens. They are the group walking independently on the one hand, they are the group with the highest increasing rate on web-surfacing through mobile phone on the other hand. This research presented the results through observation, group discussion and questionnaire on teens distracted walking by e-devices in Shanghai, which is the first of its kind in China.

Methods 8250 observations of teens in 8 communities in fall 2014 and spring 2015 were recorded. Observers conducted two morning and afternoon sessions at cross roads, on different days but at the same time, on regular school days in good weather. Each observer focused on one crossing location. 8 focus group discussions were conducted among 176 teens and 1000 questionnaires were completed by teens in 8 communities.

Results $50 \%$ of teens reported use of e-devices while walking in the questionnaire. Reported by observers at cross roads, 240 out of 8250 teens were observed distraction by e-devices while crossing in a quick traffic flow with a car passing by every 2.7 seconds. The distraction rate in the afternoon is 1.9 times vs morning. The most frequent forms of distraction were headphones $42.5 \%$, texting $22.5 \%$, game $15.8 \%$, and talking on the phones $(10.4 \%)$. During focus groups, $86.6 \%$ of teens perceived either senior or junior groups at greater risk. Safety talks on walking with e-devices with parents is in need among teens.

Conclusions Teens represent a growing proportion of road traffic victims. Prevalance use of e-devices among teens proposed growing risk. Evidence-based intervention is strenuous in need. 